\title{
On the Role of Chromium in Dynamic Transformation of Austenite
}

- K. Chadha,

- Davood Shahriari,

- C. Jr. Aranas,

- Louis-Philippe Lapierre-Boire \&

- Mohammad Jahazi

Metals and Materials International volume 25, pages 559-569 (2019)

- DOI https://doi.org/10.1007/s12540-018-00227-6 


\title{
On the Role of Chromium in Dynamic Transformation of Austenite
}

\author{
K. Chadha ${ }^{\mathrm{a}^{*}}$, Davood Shahriari ${ }^{\mathrm{a}}$, C. Jr. Aranas ${ }^{\mathrm{b}}$, \\ Louis-Philippe Lapierre-Boire ${ }^{\mathrm{c}}$, Mohammad Jahazi ${ }^{\mathrm{a}}$ \\ ${ }^{a}$ Department of Mechanical Engineering, École de Technologie Supérieure, \\ 1100 Notre-Dame West, Montreal, Quebec, Canada H3C 1K3 \\ ${ }^{\mathrm{b}}$ Department of Mechanical Engineering, University of New Brunswick \\ Fredericton, NB, E3B 5A3, Canada \\ ${ }^{\mathrm{c}}$ Finkl Steel - Sorel, 100 McCarthy, Saint-Joseph-de-Sorel, QC, J3R 3M8, Canada \\ *Corresponding author. Email: kchadha1987@gmail.com
}

\begin{abstract}
The effect of Chromium (Cr) on the dynamic transformation (DT) of austenite to ferrite at temperatures up to $430{ }^{\circ} \mathrm{C}$ above $\mathrm{Ae}_{3}$ was studied in a medium-carbon low-alloy steel. Hot compression tests were performed using Gleeble $3800^{\circledR}$ thermomechanical simulator followed by microstructural examinations using electron microscopy (FESEM-EBSD). Driving force calculation using austenite flow stress and ferrite yield stress on an inverse absolute temperature graph indicated that $\mathrm{Cr}$ increases the driving force for the transformation of austenite to ferrite; however, when the influence of stress and thermodynamic analysis are taken into account, it was observed that $\mathrm{Cr}$ increases the barrier energy and therefore, emerges as a barrier to the transformation. An analysis,
\end{abstract}


based on lattice and pipe diffusion theories is presented that quantifies the role of stress on the diffusivity of $\mathrm{Cr}$ and is compared with other the main alloying elements such as $\mathrm{C}$, $\mathrm{Si}$ and $\mathrm{Mn}$ and its impact, positive or negative, on the DT barrier energy. Finally, a comparison is made on the differential effects of temperature and stress on the initiation of DT in medium-carbon low-alloy steels.

\section{Key words}

Dynamic transformation, Medium-carbon low-alloy steel, Phase transformation, Stress induced diffusion, As-cast structure.

\section{Introduction}

Generally, the transformation of ferrite at temperatures lower than $\mathrm{Ae}_{3}$ depends upon various deformation conditions such as deformation temperature, prior austenite grain size, strain rate, precipitates, and so forth. The occurrence of dynamic transformation (DT) of austenite to ferrite at temperatures above $\mathrm{Ae}_{3}$ was first reported by Yada et al. [1,2]. They deformed Fe-C alloys using torsion testing at temperatures above $\mathrm{Ae}_{3}$ temperature. Using in-situ X-ray examinations and microstructure, it was confirmed that ferrite was dynamically formed during hot deformation. In recent years, this field has attracted a considerable attention and various research have been reported on DT of austenite to ferrite in steels [3-5], and also on other materials, such as titanium alloys, where deformation-induced transformation from a soft to hard or hard to soft phase occurs $[6,7]$.

To date, several models have been proposed to account for the phenomenon of DT above the $\mathrm{Ae}_{3}$ temperatures in steels. Hanlon et al. [8] and Ghosh et al. [9] reported that increase in Gibbs energy during deformation was responsible for DT, and related it to the stored energy from dislocation generation during hot deformation above the $\mathrm{Ae}_{3}$ temperature. However, it was later observed that transformation was initiated at the critical strain levels of $\sim 0.1$, at which the dislocation-based model did not prove significant to justify the description of the phenomenon. 
Recently, Aranas et al. [10] proposed that the driving force for the transformation can be calculated from the flow stress difference between the strain hardened austenite and the yield stress of Widmanstätten ferrite. In their model, the chemical free energy difference between austenite and Widmanstätten ferrite, the lattice dilatation work, and shear accommodation work associated with the transformation, were opposing factors to the driving force. On the role of alloying elements, they found that $\mathrm{Si}$ increased the driving force, whereas Mn decreased the net driving force. Despite extensive research on effect of alloying element on DT kinetics, the role of $\mathrm{Cr}$ on DT as an important alloying element in medium-carbon steels has not been discussed. Moreover, the role of stress on solute mobility has not been fully treated in the existing DT models while, this aspect appears to be of significant importance [11]. The present work inscribes in this context and has for main objective to study the effect of $\mathrm{Cr}$ on the driving force and barrier energy to DT of austenite to ferrite. The analysis will then be expanded to compare and evaluate the diffusion distance and diffusivity of $\mathrm{Cr}$ with other elements such as $\mathrm{C}, \mathrm{Si}$ and $\mathrm{Mn}$, while considering the stress involved during hot deformation and provide more insight into the driving force and barrier energy theories of DT.

\section{Materials and Methods}

The material used for the current investigation was an as-cast medium-carbon low-alloy steel (Labelled as, "HCr (High Chromium) alloy steel"). In order to evaluate the effect of addition of Cr, Low Cr "LCr (Low Chromium) alloy steel" with 0.1 wt. \% $\mathrm{Cr}$ and remaining alloying elements in same wt. \% as $\mathrm{HCr}$ alloy steel, was considered. The detailed compositions of both alloys are shown in Table 1. The specimens of $\mathrm{HCr}$ alloy steel were provided by Finkl Steel-Sorel Forge, Sorel, Quebec, Canada. Cylindrical specimens were machined with a diameter of $10 \mathrm{~mm}$ and a height of $15 \mathrm{~mm}$. Hot compression tests were conducted based on the ASTM E209 standards with Gleeble $3800^{\circledR}$ thermomechanical simulator. The samples were heated at $2{ }^{\circ} \mathrm{C} \mathrm{s}^{-1}$ till $1260{ }^{\circ} \mathrm{C}$ and then held $300 \mathrm{~s}$ for homogenization. The specimens were then cooled to

their deformation temperatures at the rate of $1{ }^{\circ} \mathrm{Cs}^{-1}$ before being compressed to a true strain of 0.8 and strain rate of $1 \mathrm{~s}^{-1}$. They were then water quenched immediately to 
preserve the deformed microstructure. The Schematic of the thermomechanical cycle is shown in Fig. 1.

To reveal the microstructure through Electron Back Scatter Diffraction (EBSD), the samples were mechanically polished, then electropolished at room temperature using a mixture of Perchloric acid and ethanol (1:9 by volume). The electropolishing was done at $25 \mathrm{~V}$ and $15 \mathrm{~s}$. EBSD characterization was performed with FEG-SEM (Carl Zeiss; Model: Supra 40) using TSL-OIM ${ }^{\mathrm{TM}}$ software. Low angle grain boundaries (LAGB's $>2^{\circ}$ ) are illustrated in red and high angle grain boundaries (HAGB's $>15^{\circ}$ ) are shown in black.

LCr alloy steel was selected to compare its properties with $\mathrm{HCr}$ alloy steel. JMatPro ${ }^{\circledR}$ Software [12] was used to calculate the flow stress analysis for LCr alloy steel, flow stress per phase and thermodynamic calculations for both alloys.

\section{Results and Discussions}

\subsection{Stress-Strain Curves}

The flow stress-strain data obtained after deformation at $1200{ }^{\circ} \mathrm{C}$ and $1150{ }^{\circ} \mathrm{C}$ and a strain rate of $1 \mathrm{~s}^{-1}$ using Gleeble $3800^{\circledR}$ thermomechanical simulator for $\mathrm{HCr}$ alloy steel and predicted results of LCr alloy steel are displayed in Fig. 2. The results reveal that the flow stress levels for both alloys increase with a decrease in the deformation temperature. Moreover, it can be seen that the flow stress is higher for $\mathrm{HCr}$ alloy steel than that of $\mathrm{LCr}$ alloy steel for both deformation temperatures, which indicates that the increase in wt. \% of $\mathrm{Cr}$ increases the mechanical properties of the alloy. However, all stress-strain curves present a hardening stage followed by a peak stress and then a stress drop of $\sim 3 \%$ indicating the occurrence of one or more softening processes in the material e.g., DRX [13] and DT. The occurrence of DT for $\mathrm{HCr}$ alloy steel has already been reported in a previous study [14] and the associated critical strain $(\sim 0.1)$ for the initiation of DT of austenite was determined. The values of the critical strain will be used in the present study for further calculations.

\section{Net Driving Force for Dynamic Transformation}


The $\mathrm{HCr}$ alloy steel contains austenite stabilizers such as $\mathrm{Mn}, \mathrm{Ni}, \mathrm{N}$ and Co $[15,16]$ (i.e. delay ferrite formation), and ferrite stabilizers like Si, Mo and Cr [16] (i.e., promote ferrite formation). The effects of $\mathrm{Mn}$ and $\mathrm{Si}$ on dynamic transformation of austenite was studied by Aranas et al. [10], who reported that Mn as austenite stabilizer significantly increased the driving force as well as the total barrier energy (TBE) for DT, while $\mathrm{Si}$ as ferrite stabilizer marginally increased the driving force but significantly reduced the total barrier energy (TBE) for the occurrence of DT. Due to this, the net effect of addition of Mn was found to be smaller as compared to that of Si. Therefore, in order to quantify the impact of $\mathrm{Cr}$ on DT, its driving force and contribution to the TBE need to be calculated.

Aranas et al. [10] calculated the driving force by plotting the austenite flow stress and ferrite yield stress against inverse absolute temperature using Wray et al. data [17]. The driving force calculation requires values of austenite flow stress at 0.1 (approximate critical strain for dynamic transformation) and ferrite yield stress at temperatures above $\mathrm{Ae}_{3}$. Since the yield stress of ferrite cannot be measured experimentally above the $\mathrm{Ae}_{3}$ temperature, the values of ferrite yield stress were predicted using JMat Pro ${ }^{\circledR}$ software flow stress per phase analysis tool.

Fig. 3 (a \& b) shows the graph of austenite flow stress and ferrite yield stress at deformation temperatures of $1200{ }^{\circ} \mathrm{C}$ and $1150{ }^{\circ} \mathrm{C}$ and strain rate of $1 \mathrm{~s}^{-1}$. The inverse of absolute temperature for $\mathrm{HCr}$ alloy steel (Fig. 3 (a)) and LCr alloy steel (Fig. 3 (b)) are plotted against the stress (MPa). The driving force for the transformation of austenite can be calculated from the diagrams by measuring the vertical distance between austenite flow stress (strain $=0.1)$ and ferrite yield stress. The results reveal that the increase of $\mathrm{Cr}$ content from 0.1 wt. $\%$ to 1.9 wt. $\%$ increased the driving force marginally by $\sim 6 \mathrm{Jmol}^{-1}$ at $1200{ }^{\circ} \mathrm{C}$ and $\sim 3 \mathrm{Jmol}^{-1}$ at $1150{ }^{\circ} \mathrm{C}$, relatively.

There are three types of barrier energies to the transformation of ferrite: 1) work done to accommodate localized shearing during transformation, 2) dilatation work imposed on surrounding austenite by the expansion of lower density ferrite, and 3) chemical free energy between phases $\left(\Delta G_{\gamma-\alpha}\right)[18]$. The work consumed in accommodating the process of shearing and the dilatation associated with ferrite 
formation was evaluated using the austenite flow stress developed at the measured critical strain of 0.1. The shear accommodation work per unit volume $w_{s}$ was calculated using the following relation [18]:

$$
w_{s}=m \times \sigma_{0.1} \times \gamma
$$

where, $\gamma=0.22$, is the shear strain involved in the transformation of austenite to ferrite [19], and $m=0.5$, the Schmid factor at which the grains are most favorably orientated with respect to the applied stress [10]. $w_{S}$ is expressed in the units of $\mathrm{Jmol}^{-1}$.

The dilatation work per unit volume, $w_{d}$, was calculated for both alloys in a similar manner as shear accommodation work. The orientation factors for both alloys were assumed to be, $\lambda=\sqrt{m}=0.707$ (for $m=0.5$ ). The factor was calculated for a dilatation strain of $3 \%$ using the relation [18]:

$$
w_{d}=\lambda \times \sigma_{0.1} \times 0.03
$$

The chemical free energy difference between phases $\left(\Delta G_{\gamma-\alpha}\right)$ was determined to be maximum at $1090{ }^{\circ} \mathrm{C}$ [14]. The maxima at $1090{ }^{\circ} \mathrm{C}$ indicates that at this temperature, the transformation of austenite to ferrite is most difficult to occur. Chemical free energy difference $\left(\Delta G_{\gamma-\alpha}\right)$ vs. temperature graph is shown in Fig. 4, for both alloys. Note that the values of the temperature in Fig. 4 is shown as deformation temperature minus $1100{ }^{\circ} \mathrm{C}$. With the addition of 1.9 wt. \% $\mathrm{Cr}$, the barrier to transformation increases by $\sim 13.7 \mathrm{Jmol}^{-1}$ at $1200^{\circ} \mathrm{C}$ and by $15.1 \mathrm{Jmol}^{-1}$ at $1150{ }^{\circ} \mathrm{C}$. It is also interesting to note that at the maxima, the increase in chemical free energy difference between $\mathrm{LCr}$ and $\mathrm{HCr}$ has maximum value i.e. $17.35 \mathrm{Jmol}^{-1}$ and this value keeps decreasing $\left(10.21 \mathrm{Jmol}^{-1}\right.$ at $\left.1300{ }^{\circ} \mathrm{C}\right)$ as the temperature nears delta ferrite formation temperature $\left(\sim 1480{ }^{\circ} \mathrm{C}\right.$ for both alloy steels). This indicates that, the effect of $\mathrm{Cr}$ addition gets reduced as the deformation temperature is increased. The decrease in the values of chemical free energy difference for both alloys with increasing temperature signifies that the barrier to DT is decreased as the deformation temperature nears delta ferrite temperature. 
The combined effect of these barriers to the transformation was calculated as the sum of $w_{s}+w_{d}+\Delta G_{\gamma-\alpha}$.

Driving force data, TBE and net driving force for both alloys, were calculated, and the results are shown in Figs. 5 ((a) \& (b)), for temperatures of $1200{ }^{\circ} \mathrm{C}$ (a) and $1150{ }^{\circ} \mathrm{C}$ (b), respectively. It can be seen from Fig. 5 (a) that the net driving force at $1200{ }^{\circ} \mathrm{C}$ is higher by $15.67 \mathrm{Jmol}^{-1}$ for $\mathrm{LCr}$ relative to that of $\mathrm{HCr}$ alloy. The decrease in the net driving force at $1200{ }^{\circ} \mathrm{C}$ per $1 \mathrm{wt} . \% \mathrm{Cr}$ is approximately $8.7 \mathrm{Jmol}^{-1}$. As the TBE is relatively low for $\mathrm{LCr}$ alloy steel as compared to that of $\mathrm{HCr}$ alloy, a higher net driving force was observed, despite of having lower driving force (Fig. 3 (b)). Similar trends are observed in Fig. 5 (b) $\left(1150^{\circ} \mathrm{C}\right)$ where, the net driving force is higher for LCr alloy steel by $22.19 \mathrm{Jmol}^{-1}$ relative to that of $\mathrm{HCr}$ alloy steel. The decrease in the net driving force at $1150{ }^{\circ} \mathrm{C}$ per $1 \mathrm{wt} . \% \mathrm{Cr}$ is approximately $12.3 \mathrm{Jmol}^{-1}$. The results clearly reveal that while the increase in wt. \% of $\mathrm{Cr}$ increases the driving force marginally, it significantly increases the TBE of the transformation of ferrite, which ultimately leads to reduction in the net driving force. Therefore, it appears that while $\mathrm{Cr}$ is a ferrite stabilizer, it finally acts as a barrier to the dynamic transformation of ferrite.

To further assess the underlying mechanisms of the effect of alloying elements on the dynamic transformation kinetics and microstructure, diffusion analysis of each element was carried out and presented in the next section.

\section{Diffusion Analysis of Alloying Elements}

It has been reported that carbon diffusivity increases by nearly 8.6 times when the temperature increases from $900^{\circ} \mathrm{C}$ to $1350^{\circ} \mathrm{C}$ [20-22] during the occurrence of DT in

medium-carbon low-alloy steels. However, the above works considered the temperature as the only variable parameter and did not account the contribution of stress on the diffusivity of the alloying elements. F. Masoumi et al. calculated the effect of stress on diffusion of Ni in Ni-based superalloy [23], where it was found that with the application of a compressive stress of about $187.5 \mathrm{MPa}$, the diffusion of $\mathrm{Ni}$ increased by 25 times. 
For this reason, the diffusion of $\mathrm{Cr}$ and its effect on the phenomenon of DT were studied below.

When analyzing diffusion coefficients, distinction must be made between lattice and pipe diffusion behaviors as they are the two most important mechanisms by which solutes transfer to or away from stacking faults [24]. Amongst these two, pipe diffusion, is probably the dominant mechanism in the diffusion of solutes [25]. The effective diffusion coefficient, given by $D_{e f f}$, can be defined as the sum of the lattice and pipe diffusion by the following equation,

$$
D_{e f f}=D_{L} f_{L}+D_{P} f_{P}
$$

Where, $D_{L}$ and $D_{P}$ are respectively lattice and pipe diffusion coefficients, $f_{L}$ and $f_{P}$ are fraction of atoms participating in the lattice and pipe diffusion processes. $f_{L}$ is given as unity and $f_{P}=7.03125\left(\frac{\sigma}{\mu}\right)^{2}[26]$.

The general expressions for calculating $D_{L}$ and $D_{P e f f}\left(D_{P} f_{P}\right)$ are provided in Table 2 , equations $8 \& 9$. In these equations, $b$ represents burgers vector $(m)$ and $\mu$ represents shear modulus (MPa). The equations for obtaining the values of $b$ (eq. 4) and $\mu$ (eq. 6) under applied stress were proposed by Ashby and Frost [27, 28]. Table 3 shows all the required variables for the calculation of $D_{L}$ and $D_{P}$ of $\mathrm{Cr}$, Si and Mn [27, 29-34] where, the diffusivity and diffusion distances calculated using Eqs. 8 and 9 for these three elements are compared with carbon values obtained from [14]. Tingdong [35] reported that the variations of lattice diffusion coefficient at a specific temperature could be considered independent of the stress level (i.e. constant). Thus, in this study, only the variations of $D_{P}$ will be considered for both temperatures.

In Figs. 6 (a-c), the influences of temperature and strain levels on diffusivity due to pipe diffusion (eq. 9) are illustrated for the three elements (carbon considered as reference) for $\mathrm{HCr}$ alloy steel. It is interesting to note that the diffusivity of $\mathrm{Cr}$ is significantly lower than that of $\mathrm{Si}$ and $\mathrm{Mn}$ at both temperatures by 800 and 200 times, respectively and $\sim 3000$ times lower than that of carbon. The results stating lower diffusivity of $\mathrm{Cr}$ and $\mathrm{Mn}$ as compared to Si corroborates well with the findings that with 
the addition of $\mathrm{Cr}$ (present research) and $\mathrm{Mn}$ [10], the barrier energy to DT increased, whereas it decreased with the addition of $\mathrm{Si}[10]$.

Fig. 7 ((a) \& (b)) shows the variation of diffusion distance, $x=\sqrt{D_{\text {Peff }} \times \frac{\varepsilon}{\dot{\varepsilon}}}(\mathrm{m})$ of Si (a), Cr (b) and Mn (b), atoms due to pipe diffusion at deformation temperatures of $1200{ }^{\circ} \mathrm{C}$ and $1150{ }^{\circ} \mathrm{C}$, respectively. The diffusion distance of $\mathrm{Cr}$ varies from $1.12 \mathrm{~nm}$ (at $\varepsilon=0.05)$ to $5.7 \mathrm{~nm}(\varepsilon=0.8)$ at $1200{ }^{\circ} \mathrm{C}$. Upon comparing the diffusion distance at a strain of 0.8 , the distance travelled by $\mathrm{Cr}$ is $\sim 20$ and $\sim 100$ times lower than that of $\mathrm{Mn}$ and $\mathrm{Si}$, respectively and $\sim 210$ times lower than that of carbon atoms. Such differences in the solute mobility will affect the overall kinetics of the transformation process. The grain boundary map for $\mathrm{HCr}$ alloy steel is shown in Fig. 8 (a) for the deformation temperature of $1200{ }^{\circ} \mathrm{C}$ and strain rate of $1 \mathrm{~s}^{-1}$, where the maximum average grain size (average grain size of only large grains of the map, marked by red circle) is $\sim 8.1 \mu \mathrm{m}$ and minimum average grain size (average grain size of small grains of the map, marked as black circle) is $\sim 2.4 \mu \mathrm{m}$. The criterion for determining ferrite or martensite is based on Kernal Average Misorientation map [11] (Fig. 8(b)) with the grains with lowest misorientation being ferrite (shown in blue) and those with the highest misorientation being martensite (shown in yellow). The diffusion distance of $\mathrm{Cr}$ and $\mathrm{Mn}$ is $\sim 400$ and $\sim 50$ times less than that of the minimum average grain size, whereas diffusion distance of $\mathrm{Si}$ is $\sim 1.5$ times less that of minimum average grain size. Thus, it can be said that elements such as $\mathrm{Cr}$ and $\mathrm{Mn}$ would not be able to diffuse out of grains (minimum or maximum average) during transformation, which further confirms that addition of $\mathrm{Cr}$ can impede the DT of austenite and the growth of DTed ferrite during further straining due to its sluggish diffusion.

The above findings on diffusivity and diffusion distances of various solutes provide a new insight regarding the driving force and barrier energy models for explaining the occurrence of DT. The model predicted that Si promotes the DT [10] whereas Mn [10] and Cr (present study) have a detrimental effect on it. This can be explained by the differences in the diffusivity of these three elements: during 
transformation, since the diffusivity of $\mathrm{Si}$ is high, the $\mathrm{Si}$ atoms can much faster diffuse out of the austenite matrix as compared to $\mathrm{Cr}$ or $\mathrm{Mn}$ atoms.

The effect of $\mathrm{Cr}$ on the initiation of DT can be calculated by calculating the strength of the austenite matrix. The strength can be determined by adding the work done for the dilatation and shear accommodation energy (Work ${ }_{\mathrm{D}}+\mathrm{SA}$ ) during the deformation. Work D+SA was calculated using the eqs. (1) \& (2), and then was compared for HCr alloy steel and LCr alloy steel and the results are reported in Fig. 9. It can be seen that, Work $\mathrm{D}+\mathrm{SA}$ for $\mathrm{HCr}$ alloy steel is higher by $8.68 \mathrm{Jmol}^{-1}$ and $7.53 \mathrm{Jmol}^{-1}$ at deformation temperatures of $1150{ }^{\circ} \mathrm{C}$ and $1200{ }^{\circ} \mathrm{C}$, respectively. This finding signifies that the increase in wt. \% of $\mathrm{Cr}$ increases the strength of austenite matrix, resulting in a higher austenite flow stress at the strain of 0.1 . The higher strength of the austenite matrix impedes the formation of Widmanstätten ferrite [18]. The strength of austenite can be correlated with the critical stress and critical strain of the initiation of DT. The critical strain used to calculate Work ${ }_{\mathrm{D}+\mathrm{SA}}$ is determined by double differentiation technique [36]. It has been reported that the double differentiation technique overestimates the critical stress and strain since it requires the formation of about $5 \%$ of the dynamic phase in order for the operation of the softening mechanism to be detectable [37]. To overcome this barrier, the critical stresses are calculated by the total barrier energy method, which provides exact moment that transformation begins.

The critical stress and strain for the initiation of DT are calculated by total barrier energy method using the following equation:

$$
E_{D F}=E_{B}
$$

where, $E_{D F}$ is the driving force and $E_{B}$ is the energy of the barrier to the transformation. By substituting the equations for the driving force and barrier energy (eqs. (1) and (2)), the above equation can be expressed as:

$$
\sigma_{c}-\sigma_{\beta-Y S}=\Delta G_{\gamma-\alpha}+w_{s}+w_{d}
$$

where, $\sigma_{c}$ is the critical stress for transformation, $\sigma_{\beta-Y S}$ is the yield stress of ferrite as calculated above. 
The critical stress calculated by eq. (5) was compared with the critical stress calculated by double differentiation equation for $\mathrm{LCr}$ alloy steel and $\mathrm{HCr}$ alloy steel at deformation temperatures of $1150{ }^{\circ} \mathrm{C}$ and $1200{ }^{\circ} \mathrm{C}$ [14]. It was found that the critical stresses obtained from total barrier energy method (eq. (5) and shown in Table 4) are significantly less as compared to the ones calculated by double differentiation method. From Table 5, it can be observed that the critical stresses for initiation of DT of austenite are lower for $\mathrm{LCr}$ alloy steel as compared to that of $\mathrm{HCr}$ alloy steel for both temperatures. Therefore, the increase in $\mathrm{Cr}$ restricts the occurrence of DT from austenite to ferrite.

\section{New Model for Dynamic Transformation}

In a study by Ghosh et al. [38] on the diffusion analysis of $\mathrm{C}$ and $\mathrm{Mn}$ during the DT of austenite, it was found that $\mathrm{C}$ atoms takes about $100 \mu$ s to diffuse a distance of $100 \mathrm{~nm}$, whereas, Mn atoms diffuse a distance of only $0.12 \mathrm{~nm}$ in the same period of time. Due to low diffusion distance of substitutional element, it was considered that the DT of austenite was a displacive phenomenon and diffusion (specifically of substitutional elements) does not play any role during the transformation. Their diffusion calculations employed only lattice diffusion coefficient parameters and the effect of stress (pipe diffusion) was not considered. In the present study, it was revealed that the diffusion distances for $\mathrm{C}\left(1.04 \times 10^{-6} \mathrm{~m}\right), \mathrm{Si}\left(0.53 \times 10^{-6} \mathrm{~m}\right), \mathrm{Mn}\left(12 \times 10^{-9} \mathrm{~m}\right)$ and $\mathrm{Cr}\left(1.7 \times 10^{-9} \mathrm{~m}\right)$ at $100 \mu \mathrm{s}\left(\varepsilon=0.1\right.$ and $\dot{\varepsilon}=1 s^{-1}, \frac{\varepsilon}{\dot{\varepsilon}}=0.1$ s or $\left.100 \mu \mathrm{s}\right)$ are much greater than those reported by Ghosh et al. [38], since the contribution of stress to the diffusional kinetics is taken into consideration. Therefore, the DT of austenite to ferrite is not only due to displacive transformation, but also includes diffusion of the alloying elements (e.g. substitutional elements like $\mathrm{Si}, \mathrm{Mn}$ and $\mathrm{Cr}$ ) as an additional contribution. The schematic representing the DT phenomenon is shown in Fig. 10. During hot deformation at temperatures of 1200 ${ }^{\circ} \mathrm{C}$ and $1150{ }^{\circ} \mathrm{C}$, the austenite matrix (Fig. 10 (a)) composed of interstitial (C) and substitutional ( $\mathrm{Si}, \mathrm{Cr}$ and $\mathrm{Mn}$ ) alloying elements is deformed by the action of stress. As the stress increases, the atoms of alloying elements like $\mathrm{C}$ (interstitial atom) and $\mathrm{Si}$ (substitutional atoms) start to diffuse from the austenite matrix, thus making the transformation easier (Fig. 10 (b)). However, elements like Cr and Mn (substitutional 
atoms), which have relatively lower diffusivity, remain in the austenite matrix, and thus make the austenite matrix stronger. This leads to an increase in the barrier energy due to shear accommodation and dilatation (Fig. 10 (c)) and thus acts to impede the DT of austenite. After shear accommodation and dilatation, the austenite matrix is converted into Widmanstätten ferrite (Fig. 10 (d)) [18]. The nucleation of Widmanstätten ferrite starts from the prior austenite grain boundary (PAGB) (Fig. 10 (e)). Due to the effect of stress w.r.t. to strain rate and corresponding diffusion of carbon (schematic adopted from [14]), the Widmanstätten ferrite starts to grow. At slow strain rates, the diffusion distance of $\mathrm{C}$ is less w.r.t. the grain size, which results in the formation of Quasi polygonal ferrite (QPF) (Fig. 10 (f)), however, at high strain rates, the diffusion distance of carbon is high w.r.t. to the grain size, which results in the formation of Widmanstätten Ferrite with long plates are formed (Fig. $10(\mathrm{~g}))$.

\section{Conclusions}

In the present work, the effect of $\mathrm{Cr}$ on the dynamic transformation of austenite was studied for two medium-carbon low-alloy steels. With the driving force model and diffusion analysis the following conclusions were drawn:

1. Increase in the wt. $\%$ of $\mathrm{Cr}$ from 0.1 to $1.9 \mathrm{wt} . \%$ in the alloy increased only marginally the driving force for ferrite formation, whereas, it significantly increased the barrier energy to the transformation, which signifies that the addition of $\mathrm{Cr}$ lowers the net driving force for the DT of austenite. The net decrease on the driving force at deformation temperatures of $1200{ }^{\circ} \mathrm{C}$ and $1150{ }^{\circ} \mathrm{C}$ per 1 wt. $\% \mathrm{Cr}$ is approximately 8.7 and $12.3 \mathrm{Jmol}^{-1}$, respectively.

2. Diffusion analysis indicated that $\mathrm{Cr}$ has the least diffusivity during hot deformation in the austenite matrix and therefore resulting in lower values of diffusion distance as compared to $\mathrm{Mn}$ and $\mathrm{Si}$. Due to this, the $\mathrm{Cr}$ atoms cannot move out of relatively large grains and thereby, impede DT phenomenon.

3. The lower diffusivity of $\mathrm{Cr}$ and $\mathrm{Mn}$ atoms result in lower diffusion out of the austenite matrix, thus acting as a barrier to the transformation through shearing and dilatation of austenite as compared to $\mathrm{Si}$, where shearing and 
dilatation of the austenite matrix is easier. Slower diffusivity leads to sluggish diffusion of $\mathrm{Cr}$ atoms resulting in stronger austenite matrix which eventually impedes DT phenomenon although $\mathrm{Cr}$ is a ferrite stabilizer.

\section{Acknowledgements}

The authors are very much grateful to Finkl Steel for providing the specimens for the present research. The authors would also like to thank National Science Engineering Research Council (NSERC) Canada for their support in the framework of a Collaborative Research and Development project (CRD). One of the authors (Kanwal Chadha) would like to acknowledge the support of ÉTS, Canada for financial support for visiting Indian Institute of Technology, Hyderabad, India for carrying out the EBSD characterization. We would also like to thank Dr. Pinaki Prasad Bhattacharjee for his valuable input to the analysis of EBSD images and Mr. Rajasekhar Reddy Salem for assisting in the post processing of EBSD data.

\section{References}

1. Matsumura, Y. and H. Yada, Evolution of Ultrafine-grained Ferrite in Hot Successive Deformation. Transactions of the Iron and Steel Institute of Japan, 1987. 27(6): p. 492498.

2. Yada, H., C.M. Li, and H. Yamagata, Dynamic $y \rightarrow \alpha$ Transformation during Hot Deformation in Iron-Nickel-Carbon Alloys. ISIJ International, 2000. 40(2): p. 200-206.

3. Ghosh, C., C. Aranas Jr, and J.J. Jonas, Dynamic transformation of deformed austenite at temperatures above the $\mathrm{Ae}_{3}$. Progress in Materials Science, 2016. 82: p. 151-233.

4. Park, N., et al., Flow stress analysis for determining the critical condition of dynamic ferrite transformation in 6Ni-0.1C steel. Acta Materialia, 2013. 61(1): p. 163-173.

5. Sun, L., et al., Effect of strain path on dynamic strain-induced transformation in a microalloyed steel. Acta Materialia, 2014. 66: p. 132-149.

6. Guo, B., et al., Dynamic transformation of Ti-6Al-4V during torsion in the two-phase region. Journal of Materials Science: p. 1-11.

7. Guo, B., et al., Reverse Transformation Behavior of Ti-6Al-4V After Deformation in the Two-Phase Region. Metallurgical and Materials Transactions A, 2018. 49(1): p. 22-27.

8. Hanlon, D.N., J. Sietsma, and S.v.d. Zwaag, The Effect of Plastic Deformation of Austenite on the Kinetics of Subsequent Ferrite Formation. ISIJ International, 2001. 41(9): p. 10281036.

9. Ghosh, C., et al., The dynamic transformation of deformed austenite at temperatures above the $\mathrm{Ae}_{3}$. Acta Materialia, 2013. 61(7): p. 2348-2362. 
10. Aranas Jr, C. and J.J. Jonas, Effect of $\mathrm{Mn}$ and Si on the dynamic transformation of austenite above the $\mathrm{Ae}_{3}$ temperature. Acta Materialia, 2015. 82: p. 1-10.

11. Chadha, K., et al., Influence of strain rate on dynamic transformation of austenite in an as-cast medium-carbon low-alloy steel. Materialia, 2018. 1: p. 155-167.

12. Saunders, N., et al., Using JMatPro to model materials properties and behavior. Jom, 2003. 55(12): p. 60-65.

13. Chadha, K., et al., Deformation and recrystallization behavior of the cast structure in large size, high strength steel ingots: experimentation and modeling. Metallurgical and Materials Transactions A, 2017. 48(9): p. 4297-4313.

14. K. Chadha, Z.A., C. Jr. Aranas, D. Shahriari, M. Jahazi, Influence of Strain Rate in Dynamic Transformation of Austenite in an As-Cast Medium Carbon Low Alloy Steel. Accepted for Publication in Materialia, 2018.

15. Song, H., et al., Effect of Austenite Stability on Microstructural Evolution and Tensile Properties in Intercritically Annealed Medium-Mn Lightweight Steels. Metallurgical and Materials Transactions A, 2016. 47(6): p. 2674-2685.

16. KOSKENNISKA, J., Austenitic stainless steel. 2014, Google Patents.

17. Wray, P.J., HIGH TEMPERATURE PLASTIC-FLOW BEHAVIOR OF MIXTURES OF AUSTENITE, CEMENTITE, FERRITE, AND PEARLITE IN PLAIN-CARBON STEELS. Metallurgical transactions. A, Physical metallurgy and materials science, 1984. 15 A(11): p. 2041-2058.

18. Jonas, J.J. and C. Ghosh, Role of mechanical activation in the dynamic transformation of austenite. Acta Materialia, 2013. 61(16): p. 6125-6131.

19. Bhadeshia, H., Material factors. ASM International, Member/Customer Service Center, Materials Park, OH 44073-0002, USA, 2002., 2002: p. 3-10.

20. Aranas Jr, C., et al., Flow Softening-based Formation of Widmanstätten Ferrite in a 0.06\%C Steel Deformed Above the Ae 3 . ISIJ International, 2015. 55(1): p. 300-307.

21. Grewal, R., et al., Formation of Widmanstätten ferrite at very high temperatures in the austenite phase field. Acta Materialia, 2016. 109: p. 23-31.

22. Jr., C.A., et al. Formation of Widmanstätten Ferrite in a C-Mn Steel at Temperatures High in the Austenite Phase Field. in Proceedings of the International Conference on SolidSolid Phase Transformations in Inorganic Materials (PTM 2015). 2015. Whistler, BC.

23. Masoumi, F., et al., On the Occurrence of Liquation During Linear Friction Welding of NiBased Superalloys. Metallurgical and Materials Transactions A, 2017. 48(6): p. 28862899.

24. Viswanathan, G.B., et al., Segregation at stacking faults within the $\gamma^{\prime}$ phase of two Nibase superalloys following intermediate temperature creep. Scripta Materialia, 2015. 94(Supplement C): p. 5-8.

25. Robinson, S.L. and O.D. Sherby, Mechanical behavior of polycrystalline tungsten at elevated temperature. Acta Metallurgica, 1969. 17(2): p. 109-125.

26. Samantaray, D., et al., Strain dependent rate equation to predict elevated temperature flow behavior of modified 9Cr-1Mo (P91) steel. Materials Science and Engineering: A, 2011. 528(3): p. 1071-1077.

27. Frost, H.J. and M.F. Ashby, Deformation mechanism maps: the plasticity and creep of metals and ceramics. 1982: Pergamon press.

28. Ashby, M.F., et al., Micromechanisms of flow and fracture, and their relevance to the rheology of the upper mantle. Philosophical Transactions for the Royal Society of London. Series A, Mathematical and Physical Sciences, 1978: p. 59-95.

29. Andersson, J.-O., et al., Thermo-Calc \& DICTRA, computational tools for materials science. Calphad, 2002. 26(2): p. 273-312. 
30. Taniguchi, Y., K. Hara, and M. Senoo, High pressure liquids and solutions. 2013: Elsevier.

31. Smitll, A.F. and R. Hales, Diffusion of Manganese in Type 316 Austenitic Stainless Steel. Metal Science, 1975. 9(1): p. 181-184.

32. Posner, E.S., EXPERIMENTS AND APPLICATIONS OF CHROMIUM DIFFUSION IN SPINEL. 2012, UNIVERSITY OF ARIZONA.

33. Beswick, C.L., R.D. Shalders, and T.W. Swaddle, Volume profile for substitution in labile chromium (III) complexes: Reactions of aqueous [Cr (Hedta) OH2] and [Cr (edta)]-with thiocyanate ion. Inorganic chemistry, 1996. 35(4): p. 991-994.

34. Béjina, F., et al., Activation volume of silicon diffusion in San Carlos olivine. Geophysical research letters, 1997. 24(21): p. 2597-2600.

35. Tingdong, X., Creating and destroying vacancies in solids and non-equilibrium grainboundary segregation. Philosophical Magazine, 2003. 83(7): p. 889-899.

36. Poliak, E.I. and J.J. Jonas, A one-parameter approach to determining the critical conditions for the initiation of dynamic recrystallization. Acta Materialia, 1996. 44(1): p. 127-136.

37. Aranas, C., et al., Determination of the critical stress for the initiation of dynamic transformation in commercially pure titanium. Scripta Materialia, 2017. 133: p. 83-85.

38. Ghosh, C., et al., Dynamic Transformation Behavior of a Deformed High Carbon Steel at Temperatures Above the Ae<sub>3</sub>. ISIJ International, 2013. 53(5): p. 900-908. 